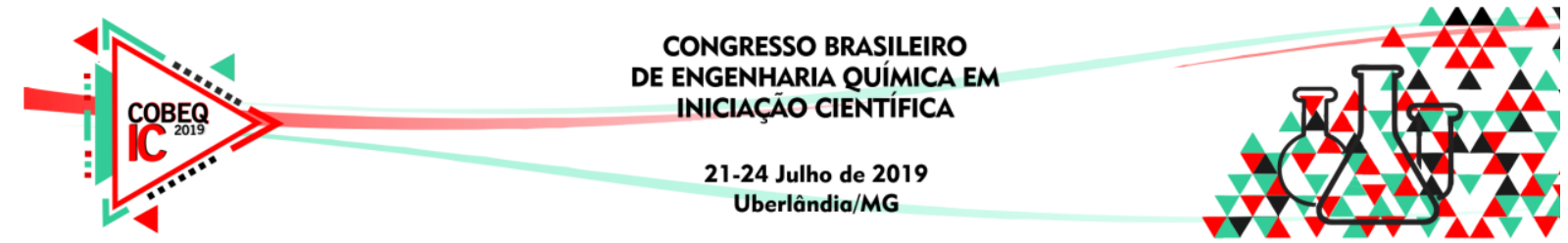

\title{
AVALIAÇÃO DA INFLUÊNCIA DA TEMPERATURA E CONCENTRAÇÃO DE ADJUVANTE NA SECAGEM POR ATOMIZAÇÃO DA FOLHA DE MARACUJÁ DOCE (Passiflora alata Curtis)
}

\author{
P. S. CAMPOS, I. O. RODRIGUES, G. J. R. SOUZA, L. F. OLIVEIRA e T. P. V. B. DIAS
}

Universidade Federal de São João Del Rei, Laboratório de Engenharia de Processos e Tecnologia

priscilla.silvacampos@yahoo.com.br

\begin{abstract}
RESUMO - A busca por produtos de melhor qualidade e livres de traços de solventes, além da preocupação com os riscos ambientais, tem levado à pesquisa de processos alternativos de secagem, mais limpos e sustentáveis. Processos de secagem por atomização são uma excelente alternativa para a secagem de alimentos com alto valor agregado, pois, através da microencapsulação, preservam as características sensoriais, nutricionais e vida útil do produto. $\mathrm{O}$ presente trabalho avaliou a influência da temperatura $\left(111\right.$ a $\left.170^{\circ} \mathrm{C}\right)$ e concentração de adjuvante (Aerosil, 21,44 a 51,55\%) na microencapsulação por spray-drying dos compostos bioativos das folhas de Passiflora alata Curtis, mais conhecida como maracujá doce, buscando uma maior preservação desses compostos. Após a secagem por atomização as partículas encapsuladas foram caracterizadas quanto à higroscopicidade, solubilidade, densidade, teor de compostos fenólicos, carotenoides, flavonoides e rendimento da secagem. No pó atomizado das folhas de maracujá doce obtivemos maior rendimento, $80,47 \%$, numa temperatura de $162^{\circ} \mathrm{C}$ e concentração de adjuvante de $25 \%$. Um ponto ótimo de secagem da folha de maracujá doce seria a uma temperatura de $165^{\circ} \mathrm{C}$ e $20 \%$ de Aerosil, para se obtiver um pó com maior teor de compostos fenólicos e a alta solubilidade.
\end{abstract}

\section{INTRODUÇÃO}

A técnica de secagem por atomização pode ser uma excelente alternativa para secagem de alimentos com alto valor agregado, pois além de aumentar a sua vida de prateleira pode vir, também, a preservar seus compostos biologicamente ativos, bem como o flavour.

O método de secagem por atomização gera produtos de alta qualidade, devido às elevadas temperaturas associadas ao curto tempo de residência do produto na câmera de secagem que resultam na elevada taxa de evaporação, dentre as suas vantagens podemos citar, controle da uniformidade da granulometria do produto, processo rápido e contínuo, maior estabilidade físico-química do produto obtido, aplicabilidade para produtos termo sensíveis e baixos custos de processo conforme Leimann et al., (2009); Oliveira \& Petrovick, (2010).

As condições de operações usadas na secagem e processamento térmico de bioprodutos podem ter considerável impacto nas propriedades e custo do produto, gerando 


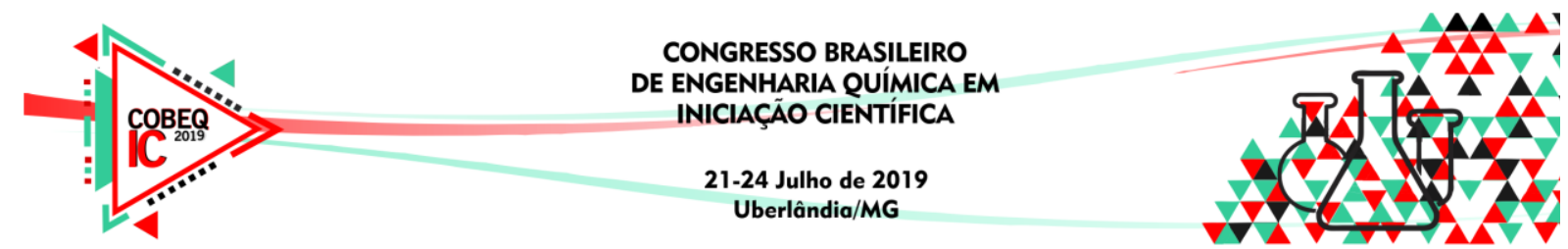

diferentes graus de perda de compostos ativos. Assim, este projeto, tem por objetivo o estudo dos efeitos das variáveis de secagem por spray-drying (temperatura e concentração de adjuvante) na folha de maracujá doce.

\section{SECAGEM POR ATOMIZAÇÃO (SPRAY-DRYING)}

Para otimização do processo de secagem variou-se os seguintes parâmetros: temperatura e concentração de Aerosil, conforme planejamento experimental baseado na Metodologia de Superfície de Resposta. Foi realizado um delineamento composto central rotacional (DCCR), sendo um fatorial completo 22, incluindo 3 pontos axiais e 3 repetições no ponto central, totalizando 11 ensaios.

\section{CARACTERIZAÇÃo DO PÓ ATOMIZADO}

Os pós atomizados em spray-drying foram caracterizados com relação à solubilidade, higroscopicidade, carotenoides, densidade, flavonoides e teor de compostos fenólicos conforme descrito a seguir. Sendo que, todas as análises realizadas em triplicata. O índice de solubilidade em água foi determinado conforme o método descrito por Cano-Chauca et. al (2005).

Para o cálculo da higroscopicidade e rendimento da secagem foi necessário à análise do teor de água, que foi determinada conforme AOAC (2002).

A higroscopicidade foi determinada de acordo com o método proposto por Tonon et. al (2009). A determinação dos carotenoides foi realizada segundo o método proposto por Higby (1962). A análise da densidade foi realizada conforme o método descrito por Jinapong et. al (2008).

A determinação de flavonoides foi realizada segundo o método proposto pela ANVISA (2010). Para a quantificação do teor de flavonoides foi realizada uma curva padrão de concentração de quercetina $(\mathrm{g})$ por absorbância $(\mathrm{nm})$.

O conteúdo de fenóis totais foi determinado pelo método de Folin Ciocalteu, segundo metodologia descrita por Singleton et. al (1999). Para a quantificação do teor de compostos fenólicos foi realizada uma curva padrão de concentração de ácido gálico (g) por absorbância (nm). Os resultados foram expressos em $\mathrm{mg} \mathrm{GAE} \mathrm{/} \mathrm{g} \mathrm{pó.}$

O rendimento dos processos de secagem foi calculado conforme a Equação 01.

Rendimento $(\%)=\frac{P_{P A}}{\left(P_{S T}\right)+\left(P_{A}\right)} \times 100$

onde, $P_{P A}$ corresponde ao peso do pó atomizado, $P_{S T}$ representa o peso dos sólidos totais presentes no extrato aquoso e $P_{A}$, o peso de adjuvante que foi utilizado na secagem. 


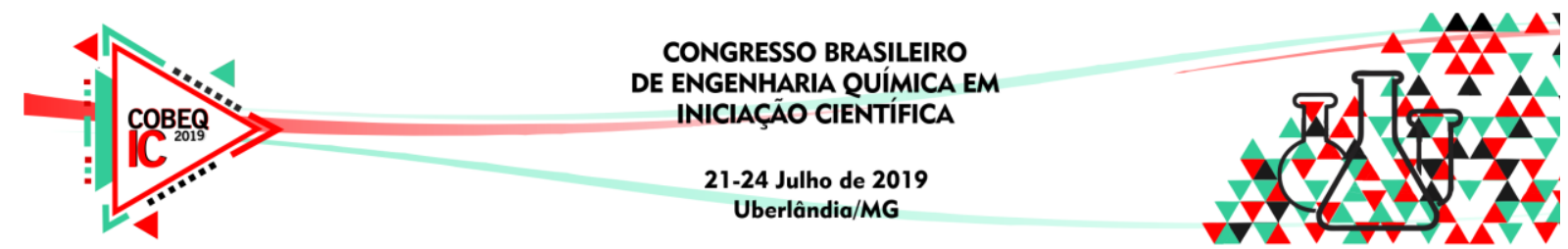

\section{RESULTADOS E DISCUSSÃO}

O maior rendimento obtido foi de $80,47 \%$, valor superior ao esperado, já que atomizadores laboratoriais que raramente fornecem rendimentos acima de 50\%, mesmo quando se utiliza grande quantidade de agentes encapsulantes coforme foi demonstrado pelo manual da Labmaq (2014).

A solubilidade do pó em água variou de 64,00 a 92,44\%, no caso em estudo é desejável uma maior solubilidade, uma vez que, o produto obtido pode ser utilizado na formulação de diversos produtos alimentícios, para enriquecimento nutricional.

Houve grande variação da densidade dos pós ficando entre 0,97 a 2,50 g/mL, o que sugere que a concentração de Aerosil e temperatura podem estar relacionadas com essa alteração significativa.

Os pós atomizados das folhas de maracujá doce obtiveram valores relativamente baixos de higroscopicidade, variando entre 13,56 a 20,14\%, o que era esperado, pois com a absorção de água as micropartículas podem mudar sua estrutura física, aderência e formar aglomerados, perdendo, assim, sua estrutura e função conforme descrito por Porras-Saavedra et al. (2015), além de tornarem-se susceptíveis a proliferação de microrganismos.

Os compostos fenólicos apresentaram teores de 50,67 a 97,94 mg GAE/g pó. Enquanto, carotenoides apresentaram valores entre 13,18 a 46,10 mg/100 g. Já os valores para o teor de flavonoides foi de 9,44 e 15,02 mg QU/g.

Para análise da significância das variáveis independentes dentro das respostas, os resultados obtidos foram submetidos à análise estatística. Considerou-se um nível de significância de $5 \%(\mathrm{p}$-valor $<0,05)$.

As variáveis dependentes, rendimento $(\mathrm{R}=0,7795)$, solubilidade $(\mathrm{R}=0,7951)$ e compostos fenólicos ( $\mathrm{R}=0,7756)$ apresentaram um bom coeficiente de determinação, indicando uma confiabilidade da análise estatística. A higroscopicidade $(\mathrm{R}=0,6255)$ e teor de flavonoides $(\mathrm{R}=0,6559)$ apresentaram um valor de coeficiente de determinação aceitável já que o processo de secagem piloto em spray-drying que envolve vários problemas operacionais como exemplo a aderência do material atomizado na parede da câmara de secagem e o controle da temperatura de saída, conforme o que foi demonstrado por Toneli et al. (2010). Já o teor de carotenoides $(0,5018)$ apresentou um baixo coeficiente de determinação, indicando que o modelo não se ajustou as dados obtidos. A temperatura não apresentou significância para nenhuma das respostas analisadas, já a concentração de Aerosil foi significativa para a solubilidade e teor de compostos fenólicos.

Analisando os coeficientes de regressão, observa-se que quanto maior a concentração de Aerosil menor a solubilidade. Isso já era esperado, já que o Aerosil é um adjuvante altamente hidrofóbico, afetando assim a solubilidade do produto conforme já foi descrito por Araújo et al. (2010).

Para os compostos fenólicos, o efeito da concentração de adjuvante foi o termo quadrático, indicando que em concentração intermediárias as amostram apresentam menor 


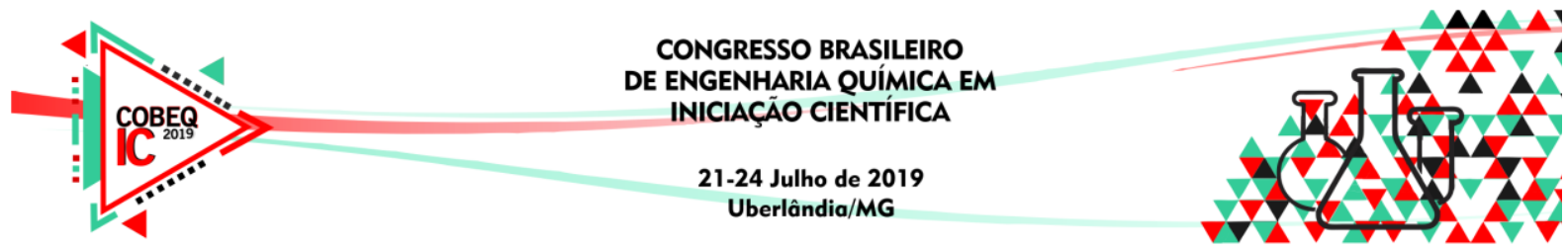

teor de compostos fenólicos. Observa-se ainda a interação entre temperatura e Aerosil foi significativa para os teores de compostos fenólicos, indicando que em menores temperaturas e maiores concentrações de Aerosil, maior é o teor compostos fenólicos no pó atomizado. $\mathrm{O}$ teor de compostos fenólicos obteve um bom ajuste de regressão ( $F_{\text {calculado maior que o }}$ $\mathrm{F}_{\text {tabelado}}$ ), assim, gerou-se a superfície de contorno (Figura 1), na qual podemos observar o efeito da concentração de aerosil e temperatura no teor de compostos fenólicos. A presença do aerosil favorece a retenção dos compostos fenólicos na cápsula, por isso em maiores concentração de adjuvante temos maiores concentração de compostos fenólicos. Apesar de maiores concentrações de aerosil, favorecerem a retenção dos compostos fenólicos, temperaturas mais elevadas degradam os compostos fenólicos que são instáveis a altas temperaturas (Rodrigues, 2011).

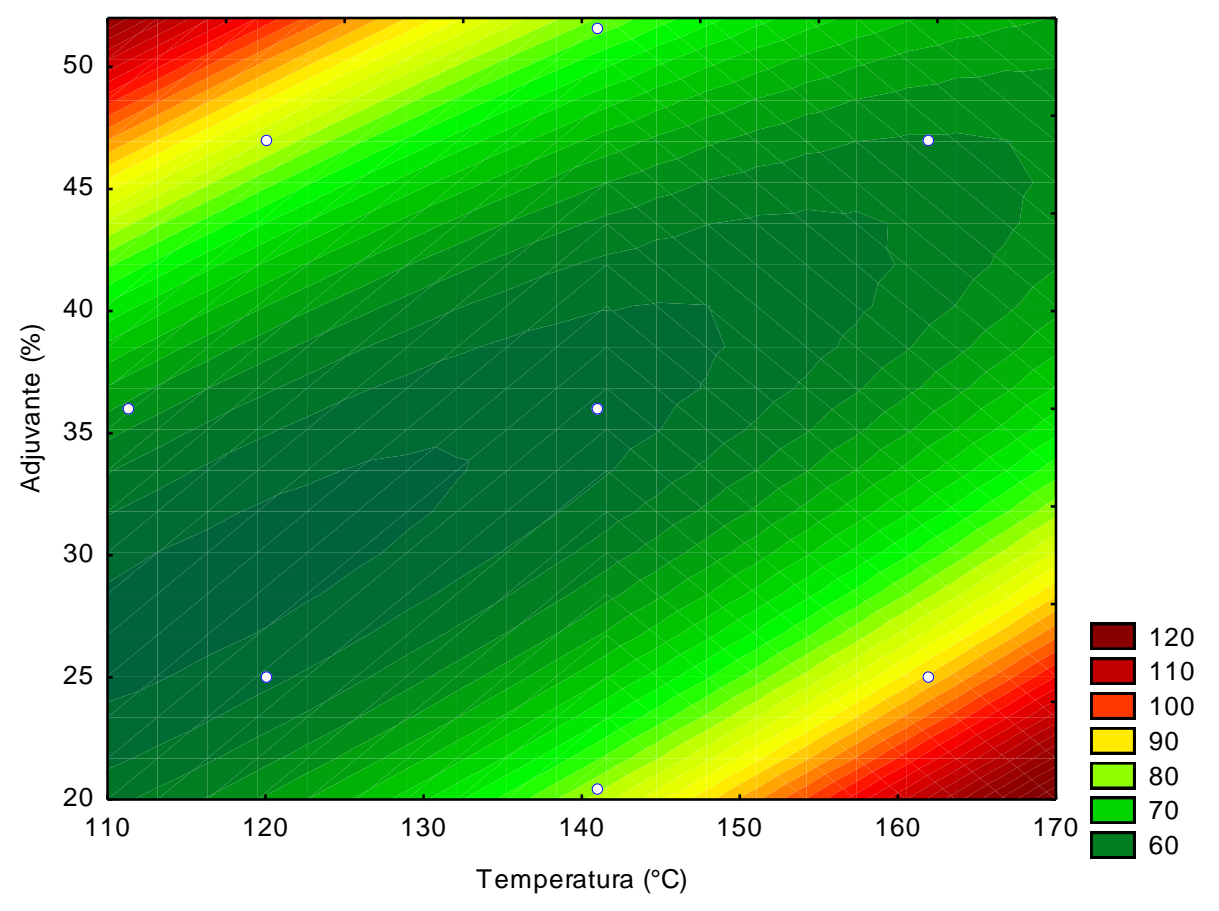

Figura 1 - Superfície de contorno para compostos fenólicos.

A variável, solubilidade, apresentou valor de $\mathrm{F}_{\text {calculado }}$ maiores que o $\mathrm{F}_{\text {tabelado }}(5,05)$, no entanto, este valor ficou muito próximo. O Aerosil é um adjuvante altamente hidrofóbico, afetando assim a solubilidade do produto conforme ARAÚJO et al. (2010), ou seja, elevadas quantidades de Aerosil levam a menores solubilidades. Para a confirmação do bom ajuste da regressão analisou-se o gráfico do valor observado versus os valores preditos, no qual foi observado que os pontos estão normalmente distribuídos em torno da reta, indicando uma linearidade considerável, ou seja, as previsões se aproximam dos valores reais, o que confirma o bom ajuste do modelo nas condições estudadas, gerando a superfície de resposta (Figura 1). 

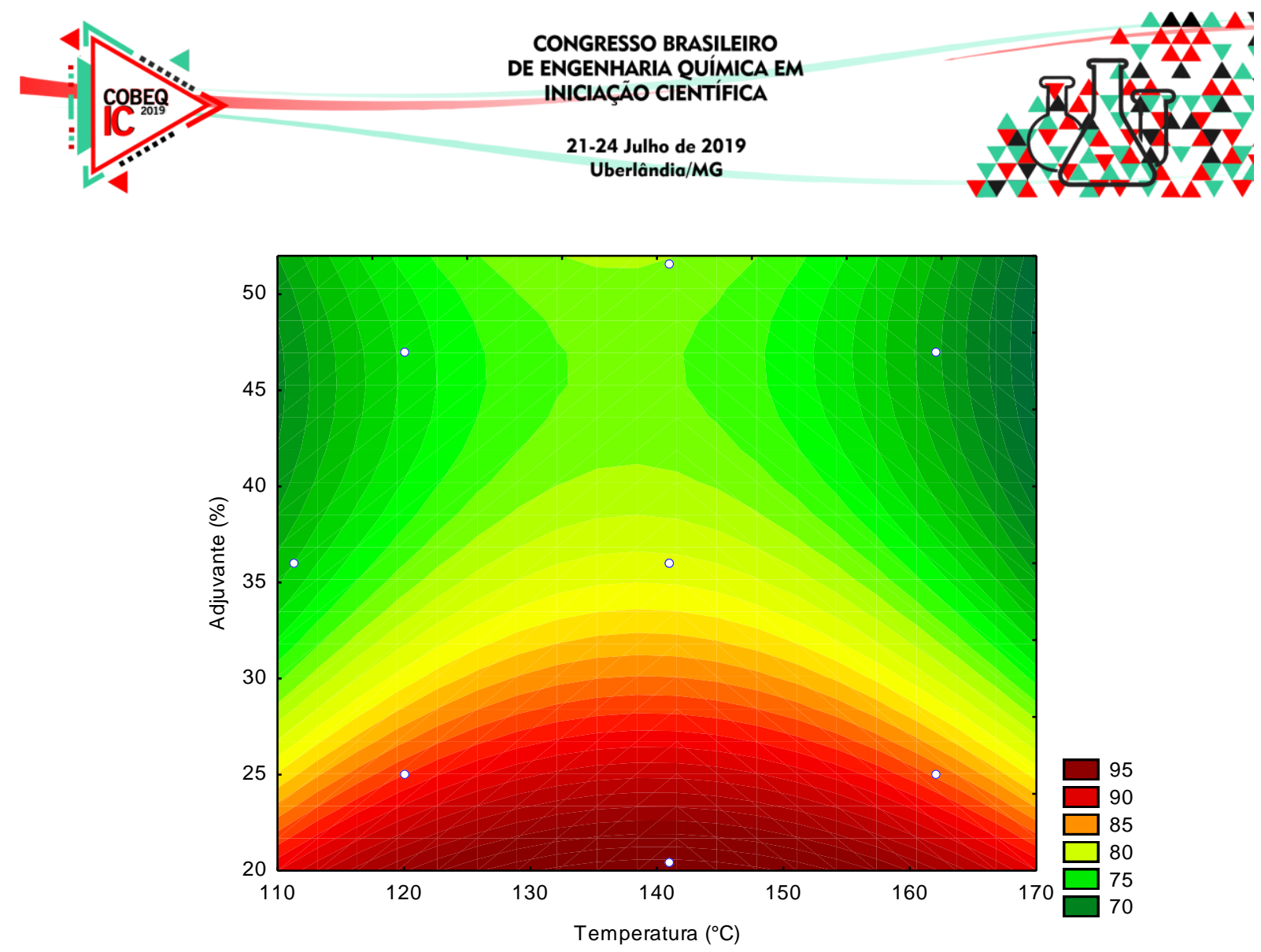

Figura 2 - Superfície de contorno para solubilidade.

O rendimento, a higroscopicidade e o teor de flavonoides não obtiveram, estatisticamente, um bom ajuste da regressão, pois os valores de $\mathrm{F}_{\text {calculado }}$ foram menores que $\mathrm{o}$ $\mathrm{F}_{\text {tabelado }}(5,05)$, não sendo possível a construção das superfícies de resposta e contorno.

\section{CONSIDERAÇÕES FINAIS}

Através deste estudo, pode-se concluir que para a secagem das folhas de maracujá doce a temperatura não se demonstrou significativa para nenhuma das respostas analisadas. Já para a concentração de Aerosil, quanto maior a concentração de Aerosil menor a solubilidade, sendo que em concentrações intermediárias de adjuvante obtiveram-se os menores teores de compostos fenólicos, indicando que a faixa ótima seria os extremos de concentração. $\mathrm{O}$ propósito geral deste trabalho era estabelecer as melhores condições de secagem por atomização em spray-drying do extrato das folhas de folhas de maracujá doce. E pode-se predizer, nas condições analisadas, um ponto ótimo de secagem a uma temperatura de, $165^{\circ} \mathrm{C}$ e $20 \%$ de Aerosil, para se ter um pó com maior teor de compostos fenólicos, e alta solubilidade.

\section{REFERÊNCIAS BIBLIOGRÁFICAS}

ANVISA; Farmacopeia Brasileira. v. 2. $5^{\text {a }}$ ed. Brasília. 2010

ARAÚJO, R. R.; TEIXEIRA, C. C. C.; FREITAS, L. A. P. The preparation of ternary solid dispersions of an herbal drug via spray drying of liquid feed. Drying Technology, v. 73, p. 134-151, 2010. 


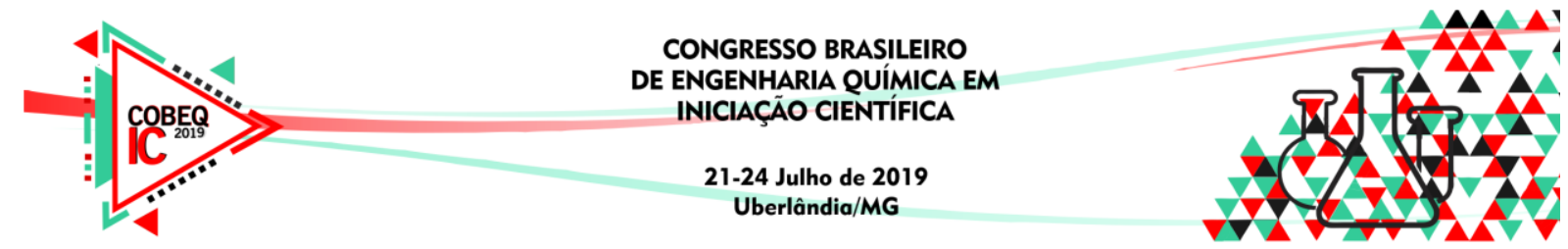

AOAC - ASSOCIATION OF OFFICIAL ANALYTICAL CHEMISTS. Official methods of analysis of Association of official Analytical Chemists International. $18^{\text {th }}$ ed. Gainsthersburg: Horwitz, 2002

CANO-CHAUCA, M.; et al. Effect of the carriers on the microstructure of mango powder obtained by spray drying and its functional characterization. Innovative Food Science \& Emerging Technologies, v. 6, n. 4, p. 420-428, 2005.

JINAPONG, N.; SUPHANTHARIKA, M.; JAMNONG, P. Production of instant soymilk powders by ultrafiltration, spray drying and fluidized bed agglomeration. Journal of Food Engineering, v. 84, n. 2, p. 194-205, 2008.

HIGBY, W. K; A simplified method for determination of the carotenoid distribution in natural and carotene fortifield Orange Juice. Journal of food Science, v. 27, p.42-49, 1962.

LABMAQ. Manual de Operação - Secador por nebulização mini "Spray dryer" Modelo MSDi 1.0. LABMAQ DO BRASIL LTDA, 2014.

LEIMANN, F.V. et al.. Antimicrobial activity of microencapsulated lemongrass essential oil and the effect of experimental parameters on microcapsules siz and morphology. Materials Science and Engineering, v. 29, p. 430-436, 2009.

OLIVEIRA, O. W.; PETROVICK, P. R. Secagem por aspersão (spray drying) de extratos vegetais: bases e aplicações. Rev Bras Farmacogn, v. 20, n. 4, p. 641-50, 2010.

PORRAS-SAAVEDRA, J.; PALACIOS-GONZÁLEZ, E.; LARTUNDO-ROJAS, L. et al. Microstructural properties and distribution of components in microparticles obtained by spray-drying. Journal of Food Engineering, v. 152, p. 105-112, 2015.

RODRIGUES, L.R.. Secagem por atomização e caracterização química de extratos de folhas de café. Dissertação de mestrado. UFLA. 2011.

SINGLETON, V. L.; ORTHOFER, R.; LAMUELA-RAVENTÓS, R. M. Analysis of total phenols and other oxidation substrates and antioxidants by means of folin-ciocalteu reagent. Methods in enzymology, v. 18, n. 299, p. 152-178, 1999.

TONELI, J.; PARK, K.; NEGREIROS, A. et al. Spray-drying process optimization of chicory root inulin. Drying Technology, v. 28, n. 3, p. 369-379, 2010.

TONON, R. V.; BRABET, C.; PALLET, D. et al. Physicochemical and morphological characterisation of açai (Euterpe oleraceae Mart.) powder produced with different carrier agents. International journal of food science \& technology, v. 31, p. 369-379, 2009. 